\title{
Protocoles d'information préopératoire: amélioration de la qualité et gestion des risques
}

\begin{abstract}
L'information aux patientes lors d'opérations gynécologiques: tel est le sujet d'un article publié dans la présente édition du BMS [1]. Ce travail, réalisé par Kessler, Ghulam, Bachmann et Haller, ainsi que l'étude publiée en 2000 et portant sur la satisfaction des patientes dans cinq cliniques de chirurgie [2], représentent un jalon important

- pour l'amélioration de la qualité de l'information donnée aux patients, et simultanément

- pour la gestion du risque en matière de responsabilité civile.
\end{abstract}

En novembre 1994, le Comité central de la FMH a recommandé aux sociétés des disciplines médicales invasives d'élaborer «petit à petit des protocoles d'information préopératoire et d'en tester l'utilité du point de vue de l'information donnée au patient». Un article à ce sujet a été publié en février 1995 dans le BMS [3] et, en juin de la même année, nous avons organisé un échange d'informations avec les sociétés médicales intéressées. Beaucoup d'entre elles ont alors commencé à ne plus voir l'information sur l'intervention chirurgicale exclusivement comme une épée de Damoclès brandie par le juge au-dessus de la médecine. La nouvelle perspective était alors et demeure la suivante: un protocole bien élaboré permet d'améliorer l'information au patient et la situation sur le plan de la responsabilité civile (gestion du risque):

- les informations et les schémas éventuels figurant dans la partie imprimée du protocole résument les aspects les plus importants de l'intervention prévue;

- la structure du protocole sert de «fil rouge» pour l'entretien consacré à l'information et permet de documenter la conversation au fur et à mesure, au moyen de quelques mots clés;

- la copie du protocole permet à la patiente de «digérer» l'entretien pour elle-même et avec ses proches;

- enfin, effet concomitant souhaité, le protocole permettra aussi aux juristes de reconstituer ultérieurement le contenu de l'entretien.
Les protocoles d'information ont été mis au point dans le cadre d'une collaboration exemplaire entre la Commission d'assurance-qualité des gynécologues, l'Organisation suisse des patients (OSP) et le service juridique de la FMH dans dix cliniques en plusieurs phases-tests. Ces protocoles portent le logo de l'OSP et celui de la FMH, attestant ainsi clairement que, selon l'état actuel des connaissances, ils représentent pour l'OSP et la FMH le meilleur compromis possible pour déterminer quelle information préopératoire peut être communiquée à la patiente et comment cette information peut être donnée.

Il ressort de l'étude actuelle que les patientes apprécient vivement cette forme d'information. Les assureursresponsabilité civile affichent également leur satisfaction. Un spécialiste confirmé des sinistres d'une grande assurance le résume ainsi: «Les protocoles de la SSGO sont super et nos clients (les hôpitaux) les remplissent la plupart du temps. Grâce à ces améliorations, un seuil critique en matière d'information du patient a été dépassé: les cas dans lesquels il existe un protocole d'information ne nous causent aucun problème.» (trad. FMH)

Une collaboration entre société médicale et organisation des patients est recommandée pour toutes les disciplines invasives importantes en vue d'optimiser les protocoles et d'assurer leur accompagnement scientifique, ce qui améliore simultanément la qualité de l'information et la situation sur le plan de la responsabilité civile.

\section{Hanspeter Kuhn, avocat, secrétaire général adjoint de la FMH}

1 Kessler M, Ghulam AT, Bachmann LM, Haller U, Keßler TM. Patientinnenzufriedenheit mit der Operationsaufklärung der Schweizerischen Gesellschaft für Gynäkologie und Geburtshilfe. Bull Méd Suisses. 2006;87(36):1607-1612.

2 Kessler M, Faisst K, Kessler W. Patientenzufriedenheit nach Operationsaufklärung mit dem Aufklärungsprotokoll der Schweizerischen Gesellschaft für Chirurgie (SGC). Bull Méd Suisses. 2000;81(34):1852-6.

3 Kuhn HP. Ärztliche Aufklärungspflicht. Bull Méd Suisses. 1995;76(8):321-6. 